\title{
Simultaneous measurement of multiparameters using a Sagnac interferometer with polarization maintaining side-hole fiber
}

\author{
O. Frazão, ${ }^{1, \star}$ S. O. Silva, ${ }^{1}$ J. M. Baptista, ${ }^{1,2}$ J. L. Santos, ${ }^{1,3}$ G. Statkiewicz-Barabach, ${ }^{4}$ \\ W. Urbanczyk, ${ }^{4}$ and J. Wojcik ${ }^{5}$ \\ ${ }^{1}$ INESC Porto, Rua do Campo Alegre 687, 4169-007, Porto, Portugal \\ ${ }^{2}$ Universidade da Madeira, Dept. de Matemática e Engenharias, Penteada, 9000-390 Funchal, Portugal \\ ${ }^{3}$ Faculdade de Ciências da Universidade do Porto, Rua do Campo Alegre 687, 4169-007 Porto, Portugal \\ ${ }^{4}$ Wroclaw University of Technology, 50-370 Wroclaw, Wybrzeze Wyspianskiego 27, Poland \\ ${ }^{5}$ Maria Curie-Skłodowska University, Lublin 20-031, Poland \\ *Corresponding author: ofrazao@ inescporto.pt
}

Received 9 July 2008; revised 14 August 2008; accepted 20 August 2008;

posted 21 August 2008 (Doc. ID 98571); published 11 September 2008

\begin{abstract}
A Sagnac interferometer with a section of a polarization maintaining side-hole fiber for multiparameter measurement is proposed. The sensor was experimentally demonstrated to be sensitive to torsion, temperature, and longitudinal strain, simultaneously. The birefringence in the investigated side-hole fiber is induced simultaneously by the elliptical shape of a germanium-doped core and by field overlap with the air holes surrounding the core. The latter effect is purely geometrical and causes high chromatic dispersion of the group birefringence in the long wavelength range, which results in a different period of spectral interference fringes. A different wavelength response is obtained for each interference fringe peak when the fiber is subjected to torsion, temperature, or longitudinal strain. A matrix equation for simultaneous measurement of the three parameters-torsion, temperature, and longitudinal strain-is also proposed. (C) 2008 Optical Society of America

OCIS codes: $\quad 060.0060,060.2370,060.2420$.
\end{abstract}

\section{Introduction}

A Sagnac interferometer or fiber loop mirror is a very attractive optical device for use in optical fiber sensing [1]. It is formed by a splice between the output ports of a directional optical coupler. A mirror is obtained when the two waves travel with identical optical paths in opposite directions, and a constructive interference is assured when the waves reenter the coupler. All the light is then reflected back into the input port, with the reflectivity limited only by the losses of the splice, fiber and coupler, while no light is transmitted to the output port. An interferometer

0003-6935/08/274841-08\$15.00/0

(C) 2008 Optical Society of America based on a fiber loop mirror can be easily obtained when a section of highly birefringent fiber ( $\mathrm{Hi}-\mathrm{Bi})$ is inserted in the loop fiber. In this case, the Hi-Bi fiber causes a difference of optical paths between the two opposite travelling waves. Besides its conceptual elegance, this configuration is attractive particularly by the fact that the periodicity of the formed spectral filter depends only on the length of the Hi-Bi fiber and not on the total length of the fiber loop [1].

In optical sensing, the Sagnac interferometer containing a section of $\mathrm{Hi}-\mathrm{Bi}$ fiber has been used in strain [2], temperature [3], liquid level [4], and displacement [5] measurement, as well as a spectral filter for fiber Bragg grating demodulation [6]. Several authors have also proposed the Sagnac interferom- 
eter for simultaneous measurement of strain and temperature when combined with a long period grating [7], or when the fiber loop contained two different Hi-Bi fibers [8].

Recently, a Sagnac interferometer based on a polarization maintaining photonic crystal fiber to measure longitudinal strain was demonstrated to be insensitive to temperature $[9,10]$. Yang et al. have proposed a fiber Bragg grating sensor interrogation technique with temperature insensitivity by using a highly birefringent photonic crystal fiber Sagnac loop filter [11]. This device was also proposed as a temperature-insensitive strain sensor $[12,13]$ and also a curvature sensor with insensitivity to strain and temperature [14].

A side-hole fiber has two air channels located in parallel to a cylindrical or an elliptical core. The side-hole fiber is a good tool for measuring pressure due to the use of the stress-optic effect when hydrostatic pressure is applied to the holes or to the cladding $[15,16]$. In this case, the air channels cause high asymmetry of the in-plane principal stress components induced by hydrostatic pressure near the core region. This type of fiber has been also applied in two different configurations for pressure sensing, namely by polarimetry [17] and by writing a Bragg grating in the elliptical core [18]. Another work analyzed the temperature sensitivity in a Sagnac loop interferometer using three types of polarization maintaining side-hole fiber [19].

In this work, a Sagnac interferometer using a section of specially designed polarization maintaining side-hole fiber is proposed for multiparameter measurements. The sensor was experimentally demonstrated to be sensitive to torsion, temperature, and longitudinal strain, simultaneously. Finally a matrix equation is proposed for simultaneous measurement of the three parameters.

\section{Experimental Results}

The side-hole fiber used in our experiments has a core dimension of $6.3 \times 2.5 \mu \mathrm{m}$ (elliptical shape), a cladding of $125 \mu \mathrm{m}$, and a jacket with acrylate coating. The difference of refractive index between the $20 \mathrm{~mol} \%$ germanium-doped core and non-doped silica cladding is 0.0264 at $633 \mathrm{~nm}$ wavelength. The longer axis of the core ellipse is perpendicular to the line that connects the centers of both holes. As shown in Fig. 1, the glass bridge between the holes is very narrow, so that the distance between the core edge and the holes edges does not exceed $2-3 \mu \mathrm{m}$. Such fiber construction causes the holes to contribute significantly to the overall fiber birefringence at longer wavelengths, at which overlap of the guided mode with air in the holes becomes significant. Similarly, like in microstructured birefringent fibers [20], this effect is responsible for high chromatic dispersion of phase $(B)$ and group $(G)$ modal birefringence. Indeed, direct measurements of $B$ carried out using lateral force method [20] shows that $B$ increases for longer wavelengths, which is characteristic for microstruc-

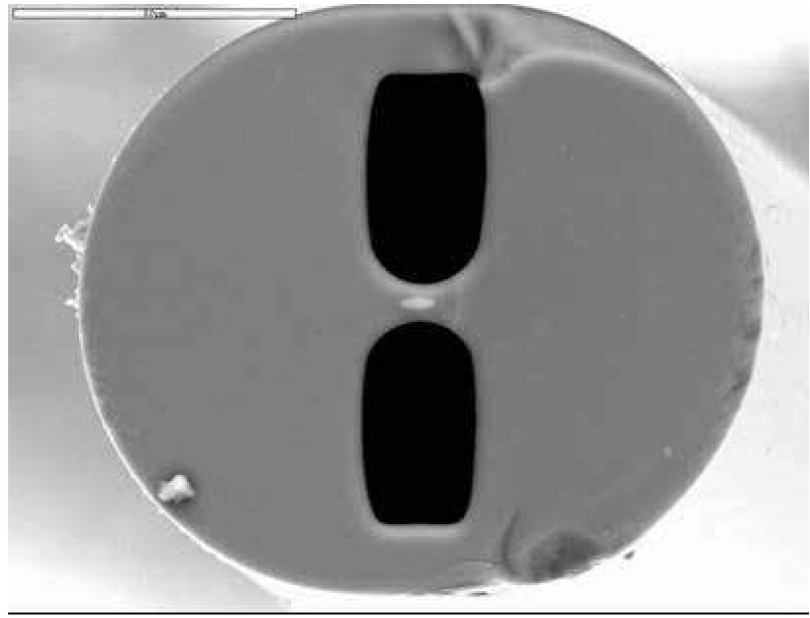

(a)

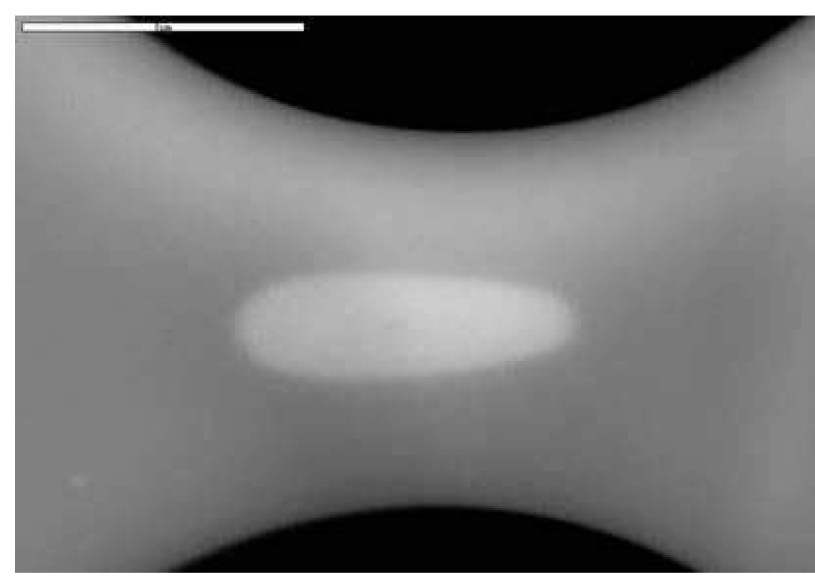

(b)

Fig. 1. SEM images of the whole cross-section of (a) the side-hole fiber and (b) the core region.

tured birefringent fibers (see Fig. 2). Such behavior of $B$ directly confirms the significant contribution of holes to the overall modal birefringence at long wavelength range. In consequence, as Fig. 2 also shows, group modal birefringence measured for the fundamental mode using the spectral interferometric method [20] strongly decreases against wavelength. High dispersion of modal birefringence in the investigated fiber makes it possible to discriminate more than one physical parameter using the same broadband source. The inset in Fig. 2 shows comparison of the group birefringence measured by the spectral interferometric method [20] and by the Sagnac interferometer. A similar result was obtained.

Figure 3(a) presents the Sagnac interferometer configuration adapted to measure torsion. It consists in a $3 \mathrm{~dB}(2 \times 2)$ optical coupler with low insertion loss, an optical polarization controller (PC), and a side-hole fiber section with a length of $0.95 \mathrm{~m}$. The interferometer is illuminated by a broadband source with a central wavelength of $1550 \mathrm{~nm}$ and the pattern fringe was read by an optical spectrum analyzer 


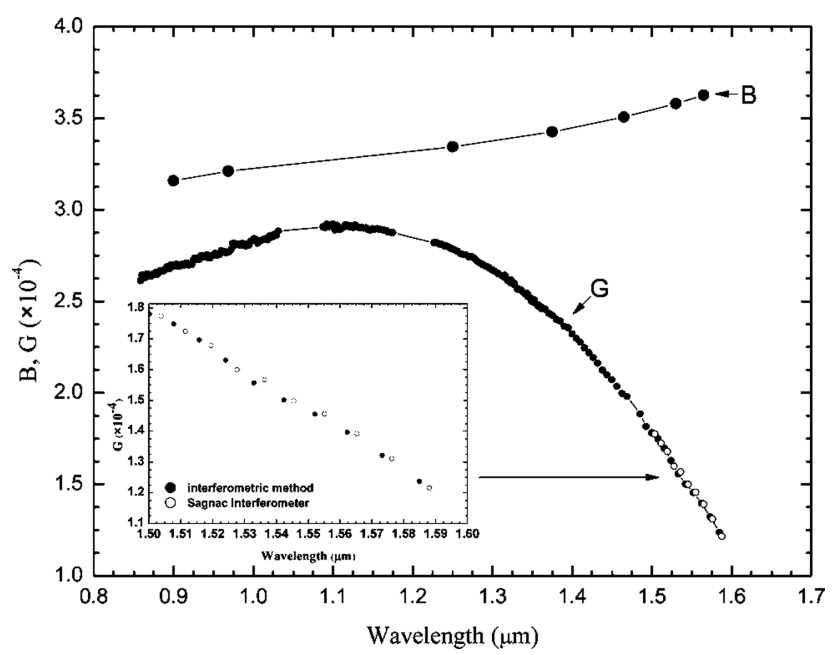

Fig. 2. Spectral dependence of phase ( $B$, blue dots) and group $(G$, black dots) modal birefringence measured in a wide spectral range. Inset figure: Comparison of the birefringence between two methods.

(OSA) with a maximum resolution of $0.05 \mathrm{~m}$. All measurements of torsion and temperature were performed with the side-hole fiber coated. Figure 3(b) shows the spectral response of the Sagnac interferometer and the four peaks (peak $i=1,2,3$, and 4) used to measure the physical parameters. The fringe pattern presents five peaks and the wavelength separation between them (period) increases with the wavelength. The group birefringence $(G)$ was calculated by the equation $G=\lambda 2 / \Delta \lambda L$, where $\lambda$ is the average wavelength and $\Delta \lambda$ and $L$, are the wavelength spacing of the fringe pattern and the length of the side-hole fiber, respectively.

For the setup of Fig. 3(a), the side-hole fiber is subjected to torsion in one fiber end, in a range of $0^{\circ}$ to $360^{\circ}$ with a constant temperature (room temperature). Figure 4 shows the displacements of the four peaks when the side-hole fiber is subjected to torsion. In this case, the separation of the peaks decreases with applied torsion, which indicates an increase of the group modal birefringence. Figure 5 shows that the birefringence presents small variation between $6.2 \times 10^{-8}$ degree $^{-1}$ to $7.1 \times 10^{-8}$ degree $^{-1}$. A second experiment was performed by subjecting the side-hole fiber to temperature without torsion. A tubular oven was used in this experiment and the results were taken when temperature was decreasing over time. The results are presented in Fig. 6 . The behavior is the same when compared with the torsion case, however the response to temperature is much greater than in conventional elliptical hole fibers. We believe that this is caused by the difference in thermooptical coefficient between the core and the cladding. It is well known that $d n / d T$ is greater in $\mathrm{GeO}_{2}$-doped glass than in pure silica. Therefore, an increase of temperature improves the confinement of the mode in the fiber core and reduces the influence of air holes on modal birefringence [21]. In consequence, the phase modal birefringence $(B)$ decreases while group modal bire-

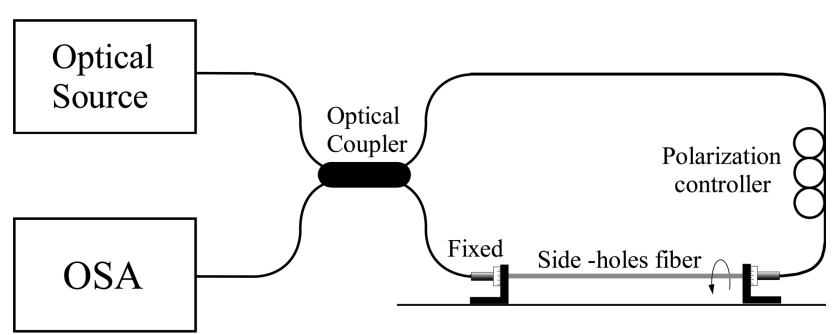

(a)

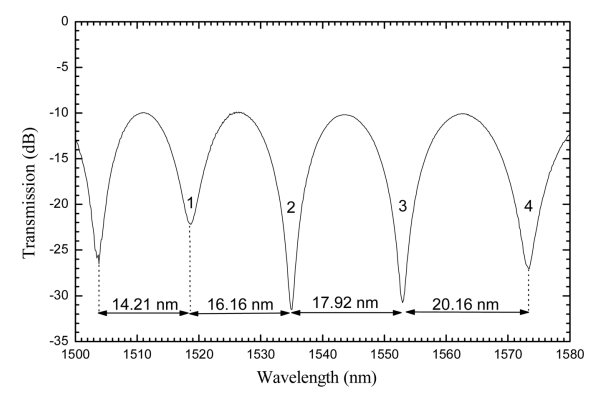

(b)

Fig. 3. (a) Experimental setup; (b) Spectral response of the Sagnac interferometer and identification of the peaks.

fringence $G$ increases against temperature (Fig. 7). The sensitivities of $G$ versus temperature for the three peaks are $11.4 \times 10^{-7} /{ }^{\circ} \mathrm{C}, 9.25 \times 10^{-7} /{ }^{\circ} \mathrm{C}$, and $8.94 \times 10^{-7} /{ }^{\circ} \mathrm{C}$.

From Figs. 4 and 6 the sensitivity coefficients when temperature and torsion variations are considered can be obtained. This is shown in Fig. 8 for peak 1 and in Fig. 3 for peak 4. The coefficients for torsion are $-0.06 \mathrm{~nm} /{ }^{\circ}$ and $-0.08 \mathrm{~nm} /{ }^{\circ}$ (peaks 1 and 4 ), while for temperature the coefficients are $-1.44 \mathrm{~nm} /{ }^{\circ} \mathrm{C}$ and $-1.97 \mathrm{~nm} /{ }^{\circ} \mathrm{C}$ (peaks 1 and 4 ).

The principle behind the sensing solutions that allows for simultaneous measurement of pairs of parameters, for instance, torsion and temperature, is the identification of two characteristics of the sensing structure that change differently accordingly to each parameter. If this happens, it is always possible to

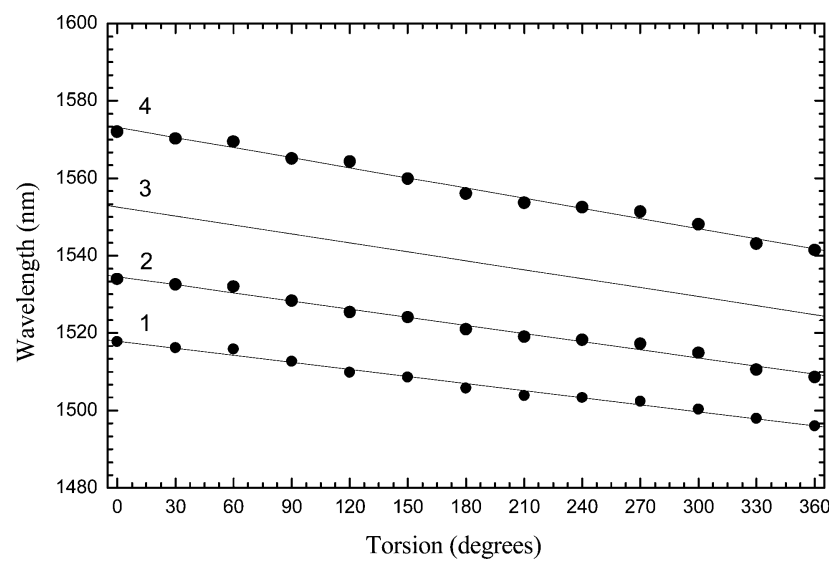

Fig. 4. Torsion response of the Sagnac interferometer. 


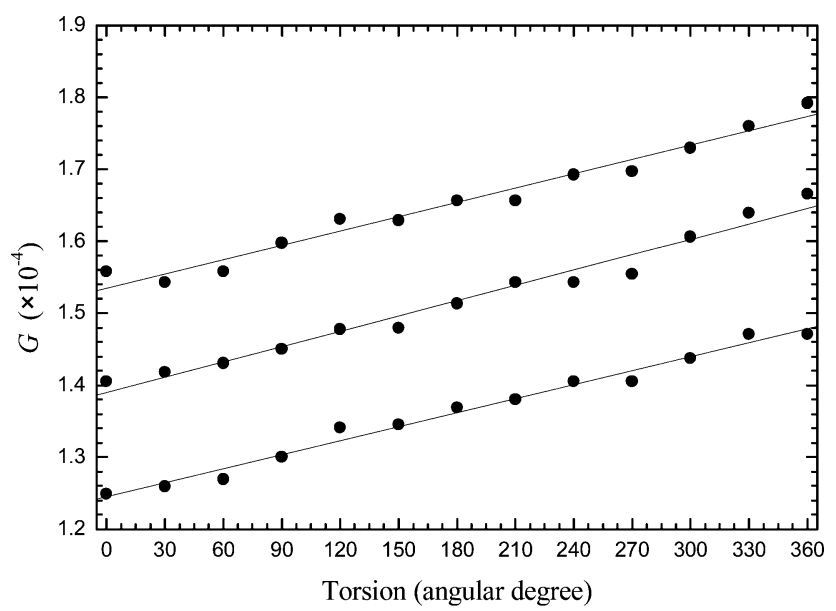

Fig. 5. Group birefringence response of the Sagnac interferometer when subjected to torsion.

write a pair of equations that give the change of each of the selected characteristics in the general situation of simultaneous actuation of the two parameters. With this procedure, a well-conditioned matrix that allows inversion can be written to obtain the actual measurand values. In the present case, the selected characteristics are the two most apart peaks (1 and 4) of the pattern fringe that appear in the Sagnac interferometer wavelength response. The wavelengths of these peaks change with variation of the applied torsion $(\Delta \tau)$ and temperature $(\Delta T)$. Therefore, it is possible to write

$$
\Delta \lambda_{\text {peak }_{i}}=K_{T i} \Delta T+K_{\tau i} \Delta \tau
$$

where $i=1,4$ addresses the two wavelength peaks considered. In such conditions, the pair of Eqs. (1) can be processed to have an explicit matrix equation for $\Delta T$ and $\Delta \tau$ :

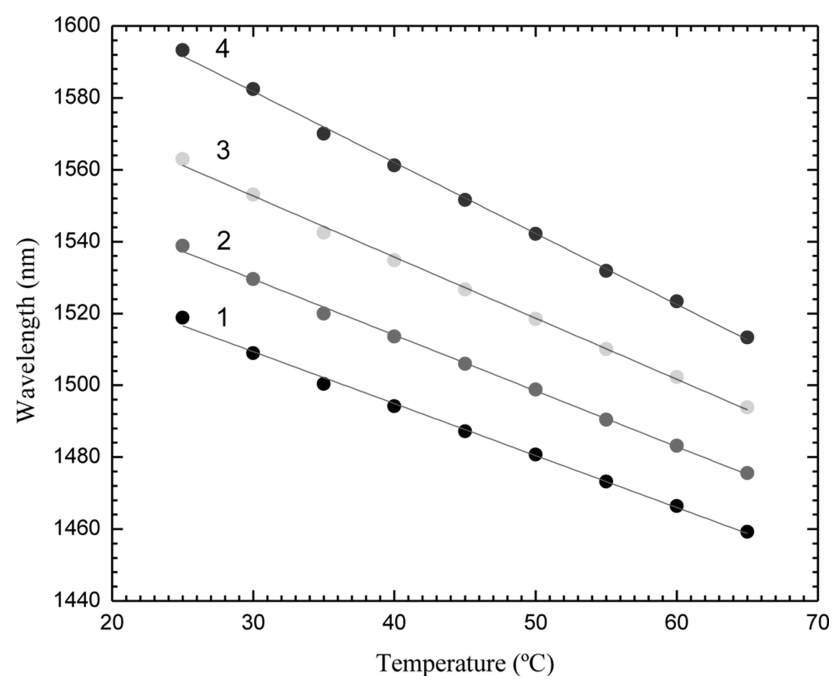

Fig. 6. Temperature response of the Sagnac interferometer.

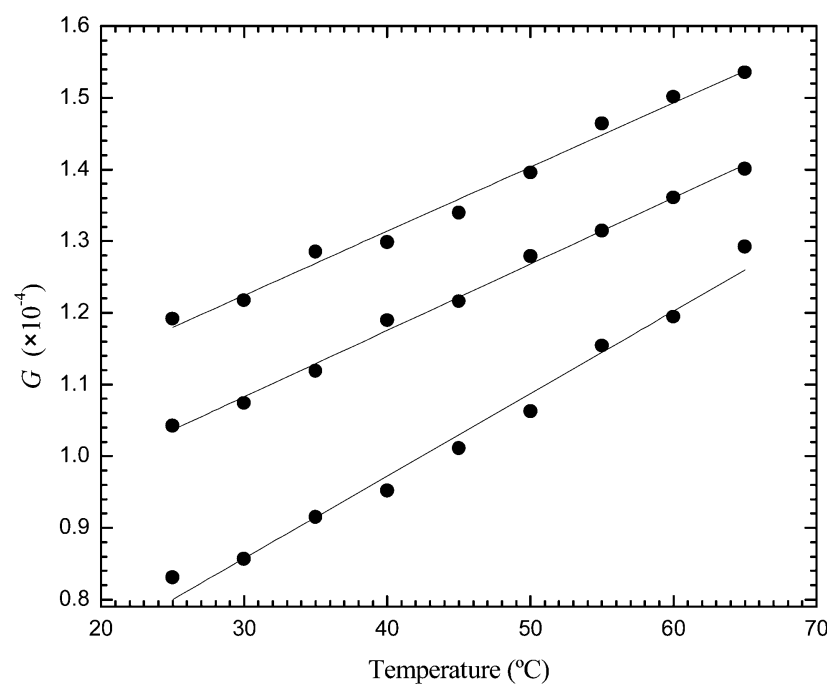

Fig. 7. Group birefringence response of the Sagnac interferometer when subjected to temperature.

$$
\left[\begin{array}{c}
\Delta T \\
\Delta \tau
\end{array}\right]=\frac{1}{D}\left[\begin{array}{cc}
K_{\tau 4} & -K_{\tau 1} \\
-K_{T 4} & K_{T 1}
\end{array}\right]\left[\begin{array}{c}
\Delta \lambda_{\text {peak } 1} \\
\Delta \lambda_{\text {peak } 4}
\end{array}\right]
$$

where $D=K_{T 1} K_{\tau 4}-K_{\tau 1} K_{T 4}$. The accuracy of the recovered $\Delta T$ and $\Delta \tau$ values increases with the increase of $D$, which in the present case depends on the difference between the sensitivity coefficients. The matrix coefficients are obtained from the experimental slopes shown in Fig. $\underline{8}$, resulting in

$$
\left[\begin{array}{c}
\Delta T \\
\Delta \tau
\end{array}\right]=-\frac{1}{3 \times 10^{-3}}\left[\begin{array}{cc}
-0.08 & 0.06 \\
1.97 & -1.44
\end{array}\right]\left[\begin{array}{l}
\Delta \lambda_{\text {peak } 1} \\
\Delta \lambda_{\text {peak } 4}
\end{array}\right]
$$

where $\Delta T$ and $\Delta \tau$ are in ${ }^{\circ} \mathrm{C}$ and in angular degrees, respectively, while the peaks wavelength shifts are in nanometers. The performance of this simultaneous measurement configuration was experimentally determined by undertaking torsion variations at a fixed temperature and the other way around, i.e., temperature variations for a constant torsion. The results are shown in Fig. 9. From these results, rms resolutions of $\pm 1.6^{\circ} \mathrm{C}$ and $\pm 5.3^{\circ}$ were determined for temperature and torsion measurements, respectively.

Figure 10 shows a real time experimental demonstration of simultaneous measurement of both physical parameters. The tubular oven was heated up to approximately $50^{\circ} \mathrm{C}$ and then gradually cooled down to $20^{\circ} \mathrm{C}$ after $20 \mathrm{~min}$. At the same time, different values of torsion $\left(360^{\circ}, 270^{\circ}, 180^{\circ}\right.$, and $\left.90^{\circ}\right)$ were applied. When the measured values for $\Delta \lambda_{\text {peak1 }}$ and $\Delta \lambda_{\text {peak } 4}$ (top of Fig. 10) were inserted into Eq. (3), it was possible to recover the actual temperature and torsion values, as can be seen in the middle and bottom pictures of Fig. 10.

The sensing configuration proposed here can discriminate other pairs of physical parameters, as is the case of temperature and longitudinal strain. For that it is necessary to characterize the sensing head behavior to strain. Figure 11 reports the results 

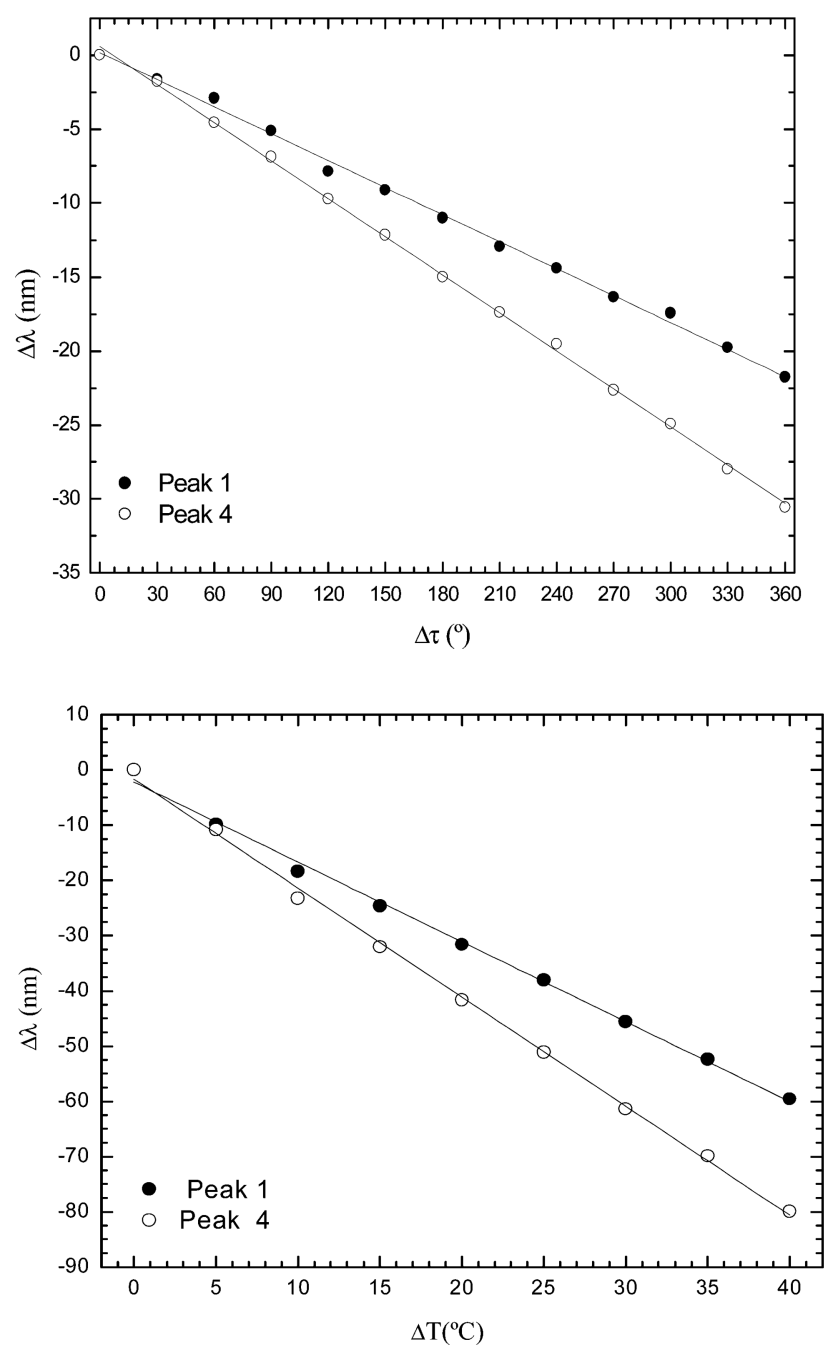

Fig. 8. Characterization of the sensing head for (a) torsion and (b) temperature.

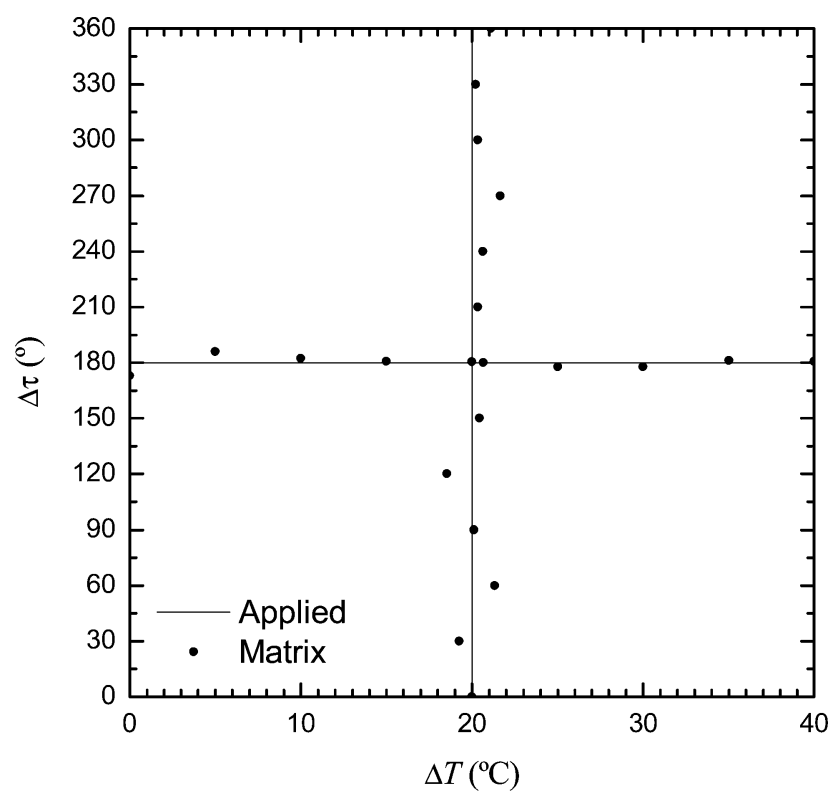

Fig. 9. Sensor output as determined by Eq. (3) for variation of applied torsion at constant temperature and variation of temperature at constant torsion.
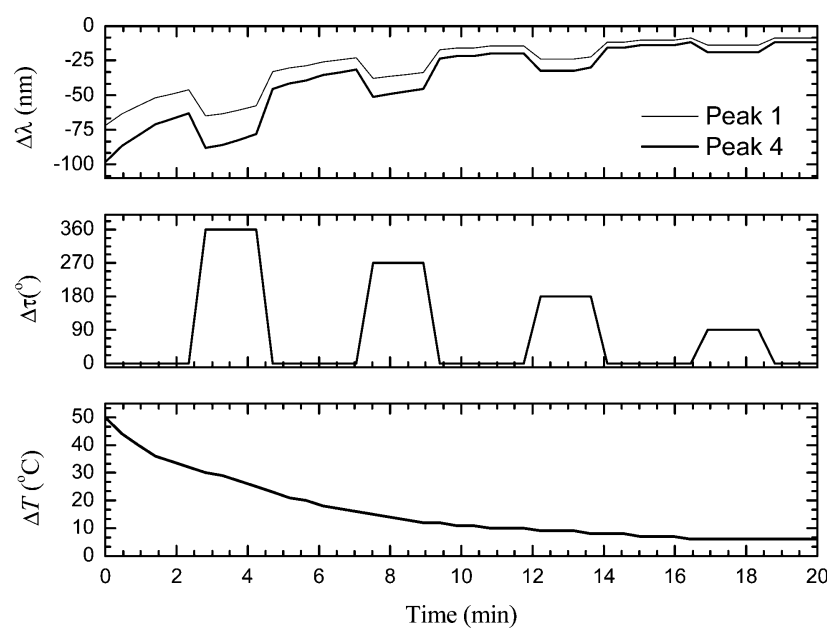

Fig. 10. Simultaneous measurement of torsion and temperature: Top, values read for $\Delta \lambda_{\text {peak1 }}$ and $\Delta \lambda_{\text {peak } 4}$ under changing temperature and torsion. Middle and Bottom, recovered values for these parameters obtained using Eq. (י).

obtained when the structure was subjected to longitudinal strain. In this case, the variation of phase and group modal birefringence is a complicated combination of confinement, geometric, and stress effects that is difficult to apprehend intuitively. Our measurements show that the overall effect brings negative slopes for all peak shifts and a small increase of group modal birefringence (Fig. 12). Considering again peaks 1 and 4 in Fig. 3 , the results in Fig. 11 allow drawing the dependences shown in Fig. 13, and from them the required wavelength-strain coefficients. Therefore, the equation for simultaneous measurement of strain and temperature becomes

$$
\left[\begin{array}{c}
\Delta T \\
\Delta \varepsilon
\end{array}\right]=-\frac{1}{1 \times 10^{-3}}\left[\begin{array}{cc}
-0.021 & 0.016 \\
1.97 & -1.44
\end{array}\right]\left[\begin{array}{l}
\Delta \lambda_{\text {peak } 1} \\
\Delta \lambda_{\text {peak } 4}
\end{array}\right]
$$

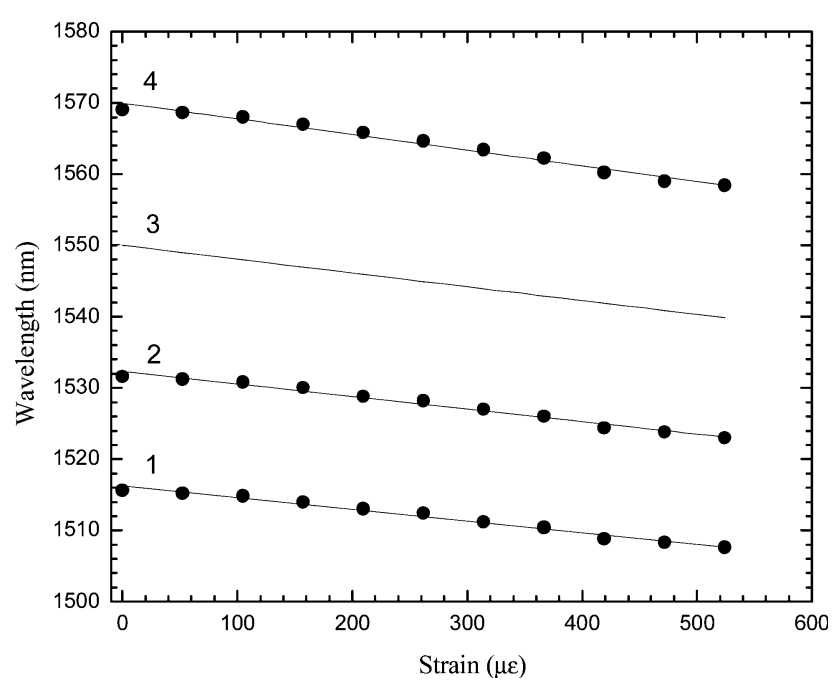

Fig. 11. Longitudinal strain response of the Sagnac interferometer. 


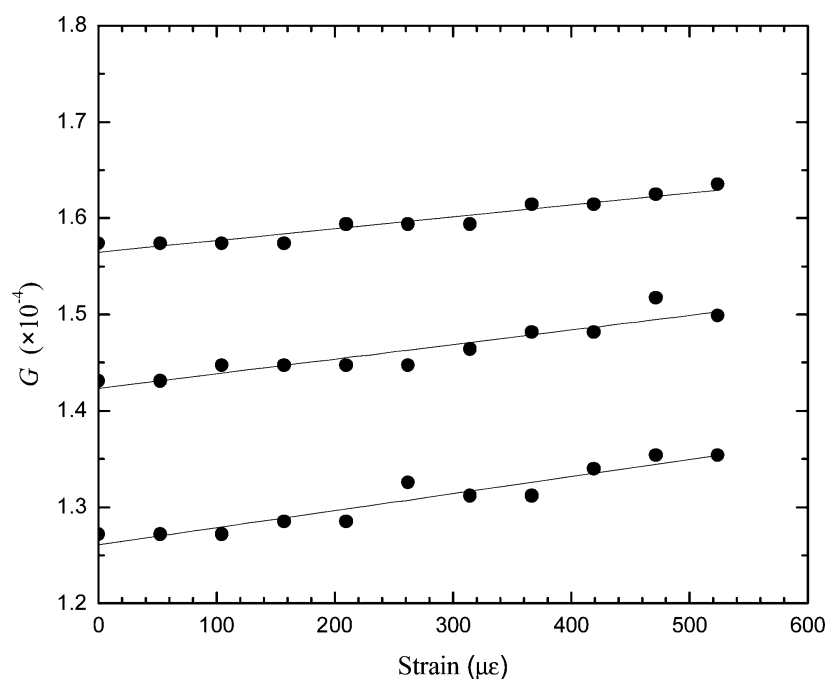

Fig. 12. Group birefringence response of the Sagnac interferometer when subjected to longitudinal strain.

The system performance for this pair of measurands was also evaluated. For that, the sensing head was simultaneously subjected to longitudinal strain and temperature changes over strain and temperature ranges of $525 \mu \mathrm{\epsilon}$ and $40^{\circ} \mathrm{C}$, respectively. From the results shown in Fig. 14, it turns out resolutions for these measurands of $\pm 10 \mu \mathrm{\epsilon}$ and $\pm 1.2 \mu \mathrm{\epsilon}$, respectively.

With this sensing structure it is feasible, in principle, to discriminate simultaneously torsion, longitudinal strain, and temperature. For that it is necessary to consider three features of the sensor transfer function shown in Fig. 3, for example the behavior with these measurands of peaks 1,2 , and 4 . For the four peaks shown in that Figure, the measurand induced wavelength change was measured, revealing in all cases a linear dependence with slopes that are summarized in Table 1.

The results in this Table show that peaks 1,2 , and 4 have different wavelength sensitivities for any one of the set of measurands (torsion, longitudinal strain,

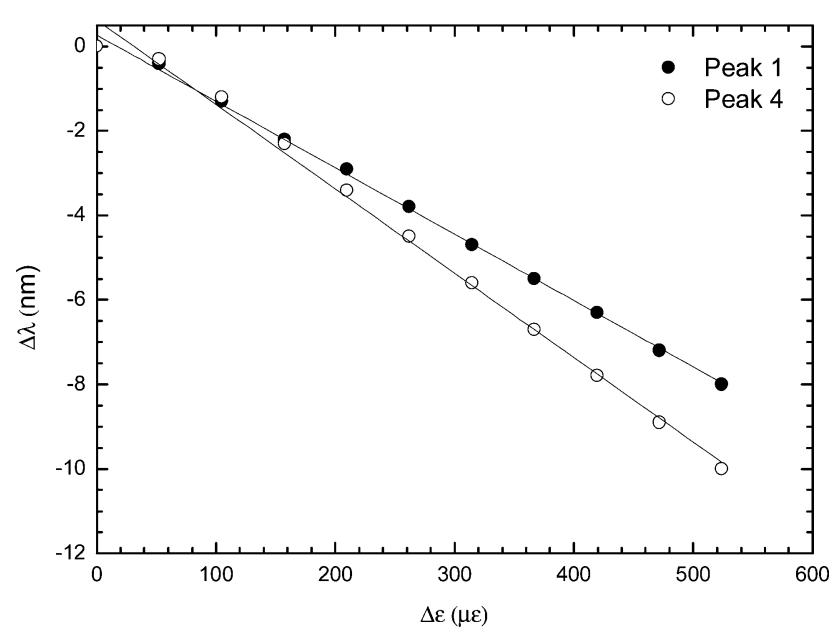

Fig. 13. Characterization of the sensing head for variations of applied longitudinal strain.

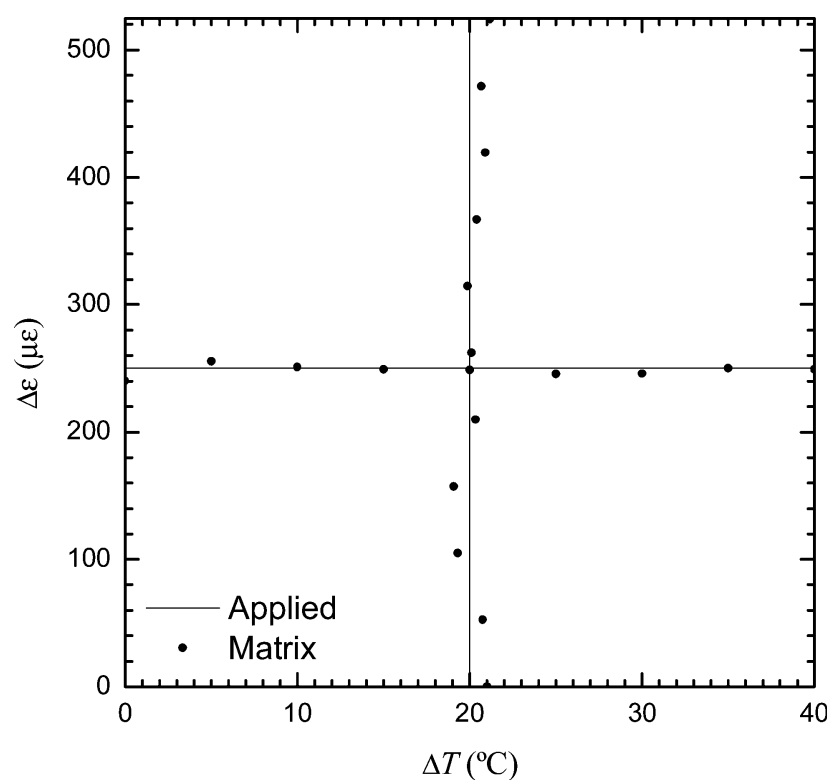

Fig. 14. Sensor output as determined by Eq. (4) for variation of applied longitudinal strain at constant temperature and temperature variation at constant strain.

and temperature). Therefore, it is possible to implement simultaneous measurement of these parameters starting with the following equation, where $i=1,2,4$ :

$$
\Delta \lambda_{\text {peaki }}=K_{T i} \Delta T+K_{\tau i} \Delta \tau+K_{\varepsilon i} \Delta \varepsilon
$$

The three equations originated by Eq. (ㅁ) can be presented in a matrix format, such as

$$
\left[\begin{array}{c}
\Delta \lambda_{\text {peak } 1} \\
\Delta \lambda_{\text {peak } 2} \\
\Delta \lambda_{\text {peak } 4}
\end{array}\right]=\left[\begin{array}{lll}
K_{T 1} & K_{\tau 1} & K_{\varepsilon 1} \\
K_{T 2} & K_{\tau 2} & K_{\varepsilon 2} \\
K_{T 4} & K_{\tau 4} & K_{\varepsilon 4}
\end{array}\right]\left[\begin{array}{c}
\Delta T \\
\Delta \tau \\
\Delta \varepsilon
\end{array}\right]
$$

being possible to be rewritten using the coefficients given in Table 1:

$$
\left[\begin{array}{c}
\Delta T \\
\Delta \tau \\
\Delta \varepsilon
\end{array}\right]=\left[\begin{array}{lll}
-1.44 & -0.06 & -0.016 \\
-1.55 & -0.07 & -0.017 \\
-1.97 & -0.08 & -0.021
\end{array}\right]^{-1}\left[\begin{array}{c}
\Delta \lambda_{\text {peak } 1} \\
\Delta \lambda_{\text {peak } 2} \\
\Delta \lambda_{\text {peak } 4}
\end{array}\right] .
$$

In this case, the resolutions using three parameters are $\pm 2.9^{\circ} \mathrm{C}$ for the temperature, $\pm 9^{\circ}$ for the torsion,

Table 1. Sensitivity Coefficients for Torsion, Temperature, and Longitudinal Strain

\begin{tabular}{lccc}
\hline & $\begin{array}{c}\text { Torsion } \\
\mathrm{nm} /{ }^{\circ}\end{array}$ & $\begin{array}{c}\text { Temperature } \\
\mathrm{nm} /{ }^{\circ} \mathrm{C}\end{array}$ & $\begin{array}{c}\text { Longitudinal Strain } \\
\mathrm{nm} / \mu \epsilon\end{array}$ \\
\hline Peak 1 & -0.06 & -1.44 & -0.016 \\
Peak 2 & -0.07 & -1.55 & -0.017 \\
Peak 3 & -0.08 & -1.70 & -0.019 \\
Peak 4 & -0.08 & -1.97 & -0.021 \\
\hline
\end{tabular}



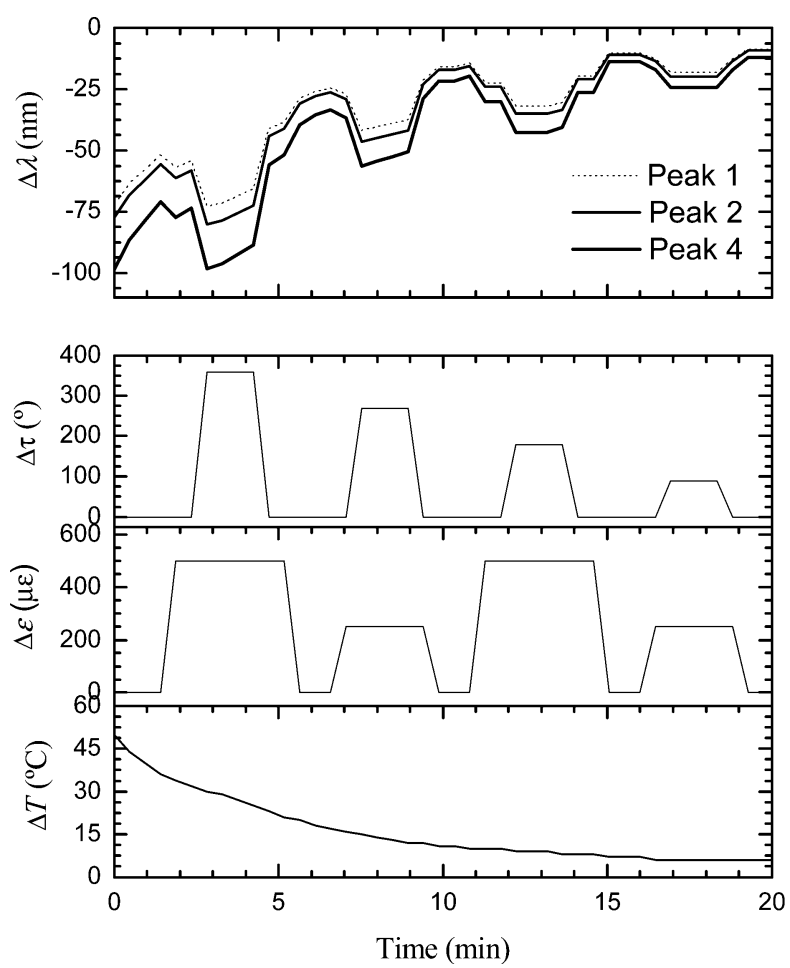

Fig. 15. Simultaneous measurement of the three parameters.

and $\pm 19 \mu €$ for the strain. The error is higher when compared with the sensing head for two physical parameters. These results are expected due to the accumulative error obtained by the three measurands [22]. Figure 15 presents the results for simultaneous measurement of the three parameters. As it can be seen, the results show the capability of the sensing configuration to measure simultaneously and independently the three physical parameters.

\section{Conclusions}

We studied the sensing characteristics of a Sagnac fiber structure that includes a length of a specially designed polarization maintaining side-hole fiber, in which birefringence is simultaneously induced by elliptical shape of the core and by mode overlap with the air holes surrounding the core. The second effect is purely geometric and induces high chromatic dispersion of group modal birefringence. The group modal birefringence of this fiber as well as its dependence upon torsion, longitudinal strain, and temperature were measured. Because of the spectral characteristics of this sensing interferometric structure, with a single side-hole fiber, the wavelength variation with these measurands, pointed out the possibility of using it for simultaneous measurement operation. This was confirmed for the pairs of torsion and temperature, longitudinal strain and temperature, and for the three parameters.

This work was supported by the COST 299Optical Fibres for New Challenges Facing the Information Society.

\section{References}

1. D. Mortimore, "Fiber loop reflectors," J. Lightwave Technol. 6, 1217-1224 (1988).

2. M. Campbell, G. Zheng, A. S. Holmes-Smith, and P. A. Wallace, "A frequency-modulated continuous wave birefringent fibre-optic strain sensor based on a Sagnac ring configuration," Meas. Sci. Technol. 10, 218-224 (1999).

3. A. N. Starodumov, L. A. Zenteno, D. Monzon, and E. De La Rosa, "Fiber Sagnac interferometer temperature sensor," Appl. Phys. Lett. 70, 19-21 (1997).

4. D. Bo, Z. Qida, L. Feng, G. Tuan, X. Lifang, L. Shuhong, and G. Hong, "Liquid-level sensor with a high-birefringence-fiber loop mirror," Appl. Opt. 45, 7767-7771 (2006).

5. Y. Liu, B. Liu, X. Feng, W. Zhang, G. Zhou, S. Yuan, G. Kai, and X. Dong, "High-birefringence fiber loop mirrors and their applications as sensors," Appl. Opt. 44, 2382-2390 (2005).

6. S. Chung, J. Kim, B-A. Yu, and B. Lee, "A Fiber Bragg grating sensor demodulation technique using a polarization maintaining fiber loop mirror," IEEE Photonics Technol. Lett. 13, 13431345 (2001).

7. O. Frazão, L. M Marques, S. Santos, J. M. Baptista, and J. L. Santos, "Simultaneous measurement for strain and temperature based on a long period grating combined with a highbirefringence fiber loop mirror," IEEE Photonics Technol. Lett. 18, 2407-2409 (2006).

8. G. Sun, D. S. Moon, and Y. Chung, "Simultaneous temperature and strain measurement using two types of high-birefringence fibers in Sagnac loop mirror," IEEE Photonics Technol. Lett. 19, 2027-2029 (2007).

9. D-H. Kim and J. Kang, "Sagnac loop interferometer based on polarization maintaining photonic crystal fiber with reduced temperature sensitivity," Opt. Express 12, 44904495 (2004).

10. C-L. Zhao, X. Yang, C. Lu, W. Jin, and M. S. Demokan, "Temperature-insensitive interferometer using a highly birefringent photonic crystal fiber loop mirror," IEEE Photonics Technol. Lett. 16, 2535-2537 (2004).

11. X. Yang, C-L. Zhao, Q. Peng, X. Zhou, and C. Lu, "FBG sensor interrogation with high temperature insensitivity by using a HiBi-PCF Sagnac loop filter," Opt. Commun. 250, 63-68 (2005).

12. X. Dong, H. Y. Tam, and P. Shum "Temperature-insensitive strain sensor with polarization-maintaining photonics crystal fiber based on Sagnac interferometer," Appl. Phys. Lett. 90, 151113-1-151113-3 (2007).

13. O. Frazão, J. M. Baptista, and J. L. Santos, "Temperatureindependent strain sensor based on a Hi-Bi photonic crystal fibre loop mirror," IEEE Sens. J. 7, 1453-1455 (2007).

14. O. Frazão, J. M. Baptista, J. L. Santos, and P. Roy, "Curvature sensor using a highly birefringent photonic crystal fiber with two asymmetric hole regions in a Sagnac interferometer," Appl. Opt. 47, 2520-2523 (2008).

15. J. Wojcik, P. Mergo, W. Urbanczyk, and W. J. Bock, "Possibilities of application of the side-hole circular core fiber in monitoring of high pressures," IEEE Trans. Instrum. Meas. 47, 805-808 (1998).

16. H. M. Xie, Ph. Dabkiewicz, R. Ulrich, and K. Okamoto, "Sidehole fiber for fiber-optic pressure sensing," Opt. Lett. 11, 333335 (1986).

17. M. N. Charasse, M. Turpin, and J. P. Le Pesant, "Dynamic pressure sensing with a side-hole birefringent optical fiber," Opt. Lett. 16, 1043-1045 (1991).

18. E. Chmielewska, W. Urbanczyk, and W. J. Bock, "Measurement of pressure and temperature sensitivities of a Bragg grating imprinted in a highly birefringent side-hole fiber," Appl. Opt. 42, 6284-6291 (2003). 
19. D. Seung Moon, B. H. Kim, A. Lin, G. Sun, Y.-G. Han, W.-T. Han, and Y. Chung, "The temperature sensitivity of Sagnac loop interferometer based on polarization maintaining sidehole fiber," Opt. Express 15, 7962-7967 (2007).

20. M. Szpulak, G. Statkiewicz, J. Olszewski, T. Martynkien, W. Urbańczyk, J, Wójcik, M. Makara, J. Klimek, T. Nasilowski, F. Berghmans, and H. Thienpont, "Experimental and theoretical investigations of birefringent holey fibers with triple defect," Appl. Opt. 44, 2652-2658 (2005).
21. J. Wojcik, B. Janoszczyk, M. Makara, K. Poturaj, W. Spytek, A. Walewski, W. Urbanczyk, and W. J. Bock, "Experimental investigation of the effect of protective coatings on temperature sensitivity of side-hole optical fibers," Proc. SPIE 3189, pp. 38-43 (1997).

22. W. Jin, W. C. Michie, G. Thursby, M. Konstantaki, and B. Culshaw, "Simultaneous measurement of strain and temperature: Error analysis," Opt. Eng. 36, 598-609 (1997). 